\title{
ORIGINAL ARTICLE \\ The effect of six weeks CoBAgi training on coordination, dynamic balance \& agility of adolescent handball players
}

\author{
Mohamad Hasif B. Mohamad Puzi ${ }^{1 \mathrm{ABCDE}}$, Lee A. Choo ${ }^{2 \mathrm{ACDE}}$ \\ Sultan Idris Education University, Tanjong Malim, Malaysia
}

Authors' Contribution: A - Study design; B - Data collection; C - Statistical analysis; D - Manuscript Preparation; E - Funds Collection.

\begin{abstract}
Purpose:

The aim of this study is to investigate the effects CoBAgi Training which apply neuromuscular training, sport specific movements that couple with perturbation variation on coordination, dynamic balance and agility among adolescent handball players.

Material: $\quad$ Quantitative randomized controlled trials quasi-experimental design with pretest and posttest evaluation used for this study. A total of 30 adolescent handball players (aged: $14.00 \pm 0.83$ years) were recruited as participants. Purposive sampling methods were used for these purposes. The participants were randomly assigned into two groups i.e. control group (CG: $n=15$ ) and experimental group (EG: $n=15$ ). EG undergone six weeks CoBAgi training while CG followed their usual training routine with supervision of the researcher, there times a week for 6 weeks. In order to measure the effect of these training on coordination, dynamic balance and agility, participants have performed Carioca Test, Star Excursion Balance Test (SEBT) and L-Run Test during pre-training and post-training session. The data were analyzed through Independent Samples t-test with $p<0.05$ "Statistical Package for the Social Sciences" (SPSS) program.

Results: $\quad$ After completed the six weeks training duration, there were significant difference identified in coordination $\left(t=-2.290, \mathrm{P}=0.030^{*}\right)$, dynamic balance $\left(\mathrm{t}=4.802, \mathrm{P}=0.000^{*}\right)$ and agility $\left(\mathrm{t}=-3.202, \mathrm{P}=0.003^{*}\right)$ between $\mathrm{EG}$ and $\mathrm{CG}$.

Conclusions: Therefore, this study revealed that CoBAgi training could improve abilities performance of coordination, dynamic balance and agility, and this suggesting that CoBAgi training may be use as rehabilitation and pre-rehabilitation training strategies in order for injury prevention plan as well as improve sports performance especially among the adolescent handball players.

Keywords: dynamic balance, coordination, agility, perturbation, pre-rehabilitation, handball
\end{abstract}

\section{Introduction}

Handball is one of the fastest team sports where this game requires high physical fitness level [1] i.e. power, speed, agility, endurance, balance, flexibility, accuracy and coordination. Undoubtedly, handball player is multitasker where he/she is a jumper, thrower and sprinter, requires good joint accelerations for landings, jumping and cutting manoeuvres. This game is physically demanding, accelerative, decelerate and intermittent in nature [2]. It also involves high degree of contact and studies revealed that record of injuries in handball sustained for every 1,000 playing hours ranging between 2.5 to 108 injuries [3]. This showed that handball players are prone to injury. Since 1999, handball rules amendments occurred and resulted with limitation for attack duration (the passive play), and converted the nature of the game to be faster [4]. Apart from that, acute and overuse injuries among adolescent have raised up in return of weakness in motor skills adaptation [5]. Thus, involvement of adolescents is handball would risk them with sport injuries.

Besides collision, individual factors would cause injuries such landing from a jump or cutting movement and, actually, with no body collision [6]. Facts reported that 115 of anterior cruciate ligament injury case in two studies i.e. $95 \%$ male and $89 \%$ female players got injured

\footnotetext{
(c) Mohamad Hasif B. Mohamad Puzi, Lee A. Choo, 202

doi:10.15561/26649837.2021.0105
}

without player-to-player contact [6]. Also, $85 \%$ of the anterior cruciate ligament injuries resulted from indirect contact or non-contact mechanisms [7]. These may be the result of sudden deceleration affected by change of direction or landing motion. For that player, especially adolescent should own a great proficiency of physical and technical abilities that can be train by manipulating some modifiable factors. Coordination, fitness level, flexibility, participation in sport-specific training, proprioception and strength are some of the modifiable elements [5]. Deficiency of these is the main contributor for non-contact injuries occurrence.

To avoid the injuries and perform well, good skills execution is needed and require excellent coordination, dynamic balance and agility interpretation. Balance shows relationship between coordination and agility. Balance is the main components of coordinative abilities which need for complex sport movements and protection against the injuries [8]. As well, enhance body balance control in positions should improve agility [9]. All the positive outcome will only be success thru manipulating the right training method with modifiable factors because such method was reported effective in sports setting [10]. In fact, these supports the need of limitless substitution number practice in handball that demands the reinforcement to design high intensity, short-duration and short-time recovery exercises such jumps, repeated sprints, contacts 
and pulling/drift actions for both conditioning and mixed (tactical and physical-based) training sessions [4]. Considering the effects of significant facts as mentioned above, the modifying of some training elements could have beneficial effects on adolescent handball players.

Numerous studies have stated that performance of the players has been improved through several training methods such as resistance training [11], neuromuscular training [12] and sport-specific training [11]. Also, there was a study that reported performance improvement through perturbation training [10]. However, some studies using combination training program in handball players on risk factors for injuries such as forward sprint-jump and vertical jump. It was reported the result of perturbationbased training led to a greater reduction in frequency of multi-step reactions and foot collisions in older adult [13]. Finding has highlighted the positive outcome for perturbation where this could be beneficial if being implement for healthy athletic community as preventive program. Perturbation simply understand as incidents that change a system state from a stable (invariant) to an unstable (variant) situation or vice versa. However, many studies only focus perturbation training on balance and proprioception for patients and there is little demonstrate its potential in a healthy, athletic population. Indeed, there is also lack of research in prevention injuries in handball. Therefore, further studies are needed to investigate the effects of combination training including perturbation training on performance.

Like mentioned before, relationship between coordination, dynamic balance and agility will play a significant impact on sports performance. The main role is contributing from balance ability which mean, improvement on it will improve coordination and agility as well. Thus, modification of training such multifaceted intervention would bring effective result [14]. With those mentioned facts, present study designed a training known as Coordination, Dynamic Balance and Agility (CoBAgi) Training on foundation with combination between specific sport skills, neuromuscular training and perturbation training. Hereby, it was hypothesized that CoBAgi Training could improve the performance of coordination, dynamic balance and agility which in return reduce the injury risk. Thus, this study aimed to investigate the effect of CoBAgi training among adolescence handball players for injury prevention.

\section{Material and Methods \\ Participants}

A total of 30 adolescence handball players of SMK Lahat enrolled in this study. The players average age, height and weight displayed as in table 1 .

\section{Research Design}

This is a quasi-experimental study with pretest and posttest evaluation involving 30 voluntary enrolled adolescence handball players, ranging from 13 to 15 years old from SMK Lahat. They were divided into experimental and control groups through random sampling method. To be selected in this study, participants need to be healthy and with at least one year free from injuries. They were excluded if they were identified with recurrent injuries or got injury during implementation of study.

Before admission to this study, participants and their parents/guardian were briefed with the study purposes, benefits, risk and parents/guardian signed the informed consents, as agreement proof for their children participation.

Participants answered screening form to make sure that only players with no injuries or free from injuries with at least one year being selected. Three testing instruments used to identify three components which are dynamic balance, coordination and agility. Participants were tested prior the intervention, and tested again after completed the intervention.

Score of pretest and posttest being compared to identify the different. Star Excursion Balance test (SEBT) was used to access dynamic balance, Carioca Test was used to access coordination and L-Run Test was used to access agility. Training duration were completed in six weeks with three sessions per week in which the training session organized at the school field. Experimental group being given CoBAgi Training as in Table 2 while control group trained as usual under the supervision of the researcher. Participants in control group was not given any special intervention which they just practice their normal training.

Table 1. Demographic Data of Experimental Group and Control Group

\begin{tabular}{lll}
\hline Variables & Experimental Group & Control Group \\
\hline Gender & 9 males/6 females & 8 males/7 females \\
\hline Age (Years) & $14.13 \pm 0.83$ & $13.6 \pm 0.91$ \\
Height $(\mathrm{cm})$ & $162.13 \pm 7.48$ & $158.0 \pm 7.06$ \\
Weight $(\mathrm{kg})$ & $51.45 \pm 7.15$ & $51.33 \pm 5.73$ \\
BMI $\left(\mathrm{kg} \div \mathrm{cm}^{2}\right)$ & $19.5 \pm 1.76$ & $20.53 \pm 1.49$ \\
\hline
\end{tabular}

NOTE: $\mathrm{cm}$ - Centimetre or centimetre is a unit of length in the metric system; kg - Kilogram; BMI - Body Mass Index; $\mathrm{cm}^{2}-\mathrm{cm}^{2}$ is part of a group of pressure units that relate pressure with weight; \pm - Values are presented as mean \pm standard error. 
Table 2. Coordination, Dynamic balance and Agility Intervention (CoBAgi Protocol)

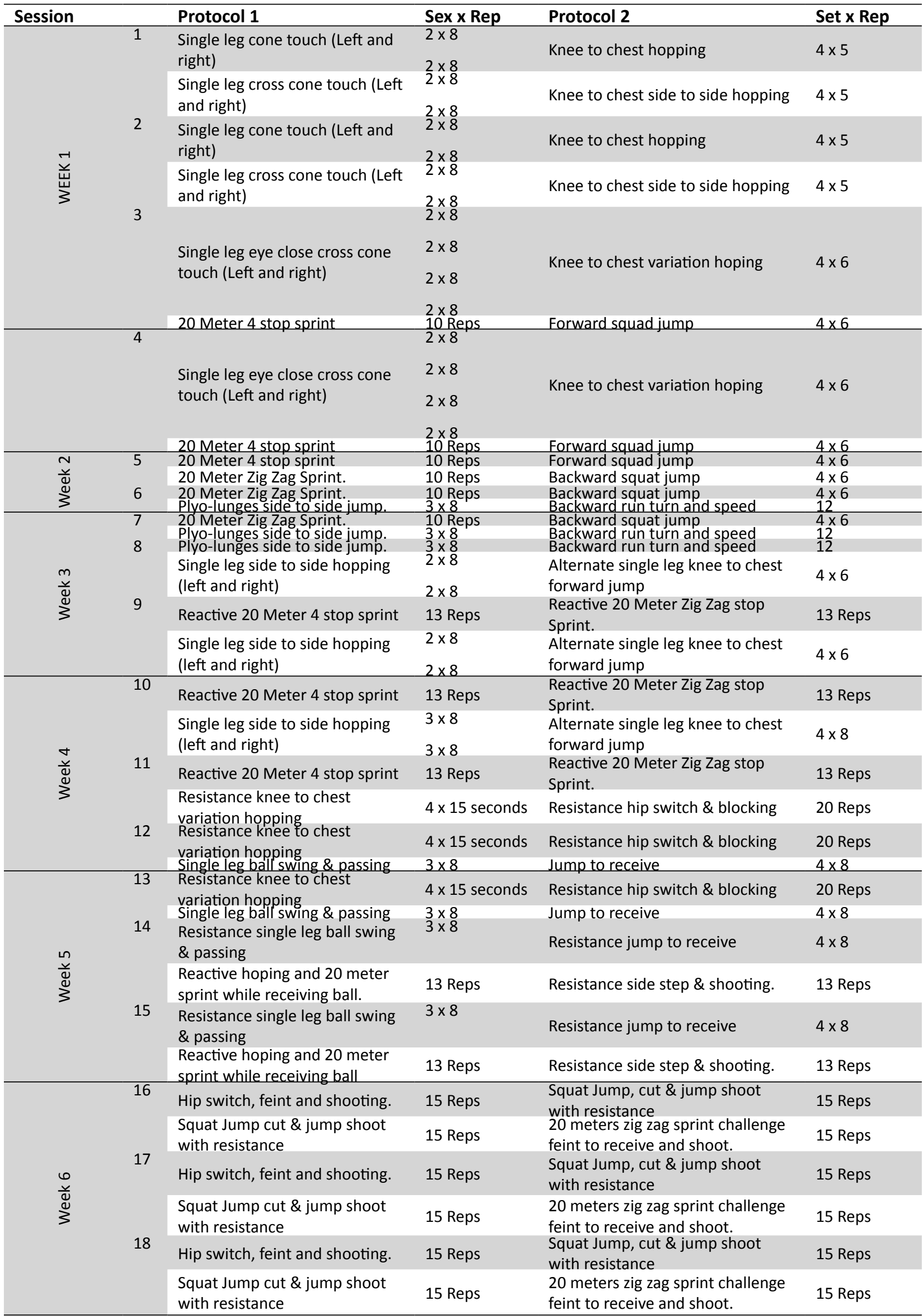

NOTE: Plyo - plyo refer to exercises in which muscles exert maximum force in short intervals of time with the goal of increasing power. 


\section{Statistical Analysis}

The recorded data were statistically processed using the IBM Statistical Package for Social Sciences version 22.0. Data were used to display as in mean, standard deviation, t-value and p-value after the analysis, the mean difference for measuring variables were statistically tested using independent sample t-test to compare the result on scores differences between pretest and posttest within group and between group. Graphs were plotted to show the changes in all related variables. All statistical tests were tested at $\mathrm{P}<0.05$.

\section{Results}

The effect of CoBAgi training in SEBT score

As demonstrated in Figure 1, there was a statistically significant difference within group in EG for SEBT score $\left(\mathrm{t}=-4.556, \mathrm{P}=0.000^{*}\right)$ where EG showed significant improvement in functional reaching score for the posttest. After six weeks with CoBAgi training, there was (6.51 $\mathrm{cm})$ improvement for functional reaching for posttest in EG from $68.07 \pm 2.98 \mathrm{~cm}$ to $74.58 \pm 2.73 \mathrm{~cm}$ (Table 3). However, $\mathrm{CG}$ indicated that there was no significant difference within group in SEBT score $(\mathrm{t}=-0.448, \mathrm{P}=$ $0.661)$ i.e. the mean score SEBT in functional reaching was from $67.18 \pm 2.44 \mathrm{~cm}$ for pretest and $67.78 \pm 2.93 \mathrm{~cm}$ for posttest (Table 3).

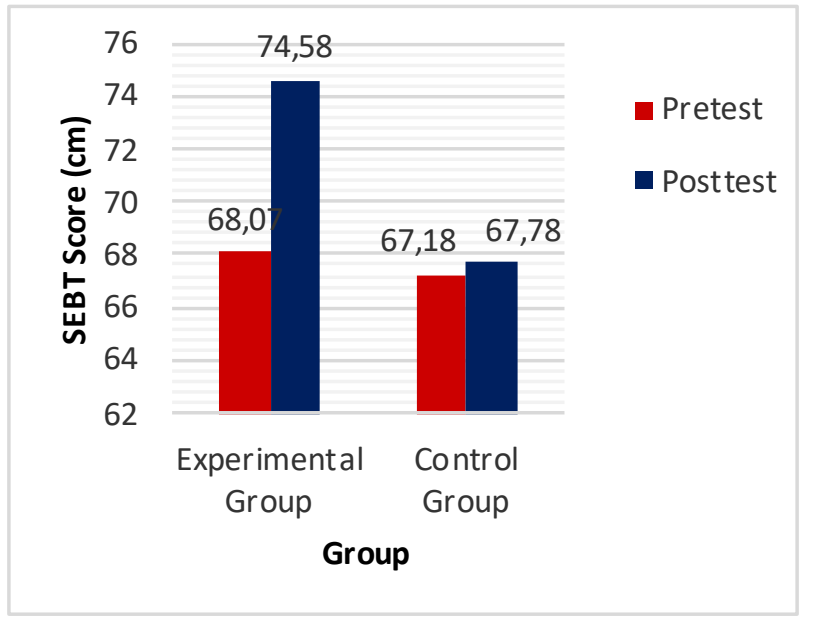

Figure 1. The Comparison Scores of Pretest and Posttest for SEBT between and within experimental group and control group after Six Weeks of CoBAgi Training
The pretest value for SEBT between score EG and CG before six weeks of CoBAgi Training $\mathrm{t}=0.653, P=0.525$ indicated subjects were from homogenous group. After six weeks of intervention, there was significant difference between EG and CG in the posttest $\left(\mathrm{t}=4.802, P=0.000^{*}\right)$ where mean score for EG in the posttest was $74.58 \pm 2.73$ $\mathrm{cm}$ and mean score for CG was $67.78 \pm 2.93 \mathrm{~cm}$ i.e. the score difference was $6.80 \mathrm{~cm}$ (Table 3).

The effect of CoBAgi training in Carioca score

As demonstrated in Figure 2, there was a statistically significant difference within pretest and posttest of EG for Carioca score $\left(\mathrm{t}=2.596, P=0.015^{*}\right)$ where EG showed significant improvement in posttest indicating after six weeks with CoBAgi training, there was reduction in time taken to complete carioca test for posttest in EG i.e. $10.60 \pm 2.02 \mathrm{sec}$. to $8.70 \pm 2.00 \mathrm{sec}$. (reduction of 1.90 sec.) (Table 4). However, CG indicated that there was no significant difference within pretest and posttest in Carioca score $(\mathrm{t}=-0.875, P=0.389)$ i.e. the mean score for time taken to complete Carioca test was pretest 10.57 $\pm 1.51 \mathrm{sec}$. and posttest $10.11 \pm 1.33 \mathrm{sec}$. (Table 4 ).

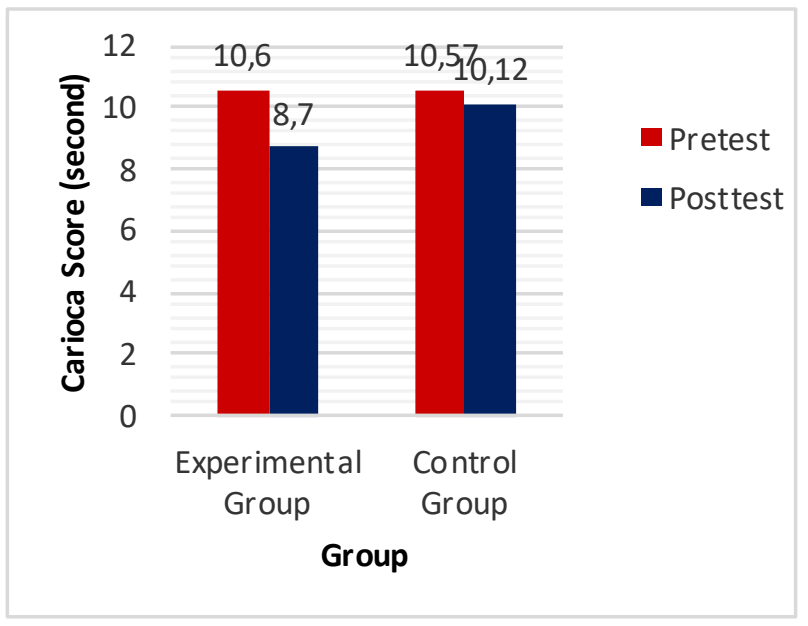

Figure 2. The Comparison Scores of Pretest and Posttest for Carioca test between and within experimental group and control group after Six Weeks of CoBAgi Training

The pretest value for Carioca test between EG and CG before six weeks of CoBAgi Training was $\mathrm{t}=0.046$, $\mathrm{p}=0.964$ indicating EG and CG were from homogenous group. After six weeks of intervention, there was significant difference between EG and CG in the posttest

Table 3. The Comparison of Pretest and Posttest Between and Within Control Group and Experimental Group in SEBT Score after Six Weeks with CoBAgi Training

\begin{tabular}{lllll}
\hline Group & Pre & Post & $t$ & $P$ \\
\hline Experimental Group & $\mu \pm S D$ & $\mu \pm S D$ & -4.556 & $0.000^{*}$ \\
Control Group & $67.07 \pm 2.98$ & $74.58 \pm 2.73$ & -0.448 & 0.661 \\
Group & & $67.78 \pm 2.93$ & & \\
$\mathrm{t}$ & 0.653 & & & \\
$P$ & 0.525 & 4.802 & & \\
\hline
\end{tabular}

NOTE: SD - Standard Deviation; \pm - Values are presented as mean \pm standard error. 
$\left(\mathrm{t}=-2.290, \mathrm{P}=0.030^{*}\right)$ where mean score for $\mathrm{EG}$ in the posttest was $8.70 \pm 2.00 \mathrm{sec}$. and mean score for CG was $10.11 \pm 1.33 \mathrm{sec}$. (i.e. the score difference for $\mathrm{EG}$ and $\mathrm{CG}$ was $1.41 \mathrm{sec}$.) (Table 4).

The effect of CoBAgi training in L-Run score

As demonstrated in Figure 3, there was a statistically significant difference within pretest and posttest of EG for L-Run score $(\mathrm{t}=2.680, \mathrm{P}=0.012 *)$ where $\mathrm{EG}$ showed significant improvement in posttest indicating after six weeks with CoBAgi training, there was reduction in time taken to complete L-Run test for posttest in EG i.e. $6.92 \pm$ $0.75 \mathrm{sec}$. to $6.23 \pm 0.69 \mathrm{sec}$. (reduction of $0.69 \mathrm{sec}$.) (Table 5). However, $\mathrm{CG}$ indicated that there was no significant difference within pretest and posttest in L-Run score $(\mathrm{t}$ $=-0.045, \mathrm{P}=0.964)$ i.e. the mean score for time taken to complete L-Run test was pretest $7.04 \pm 0.74 \mathrm{sec}$. and posttest $7.05 \pm 0.74 \mathrm{sec}$. (Table 5).

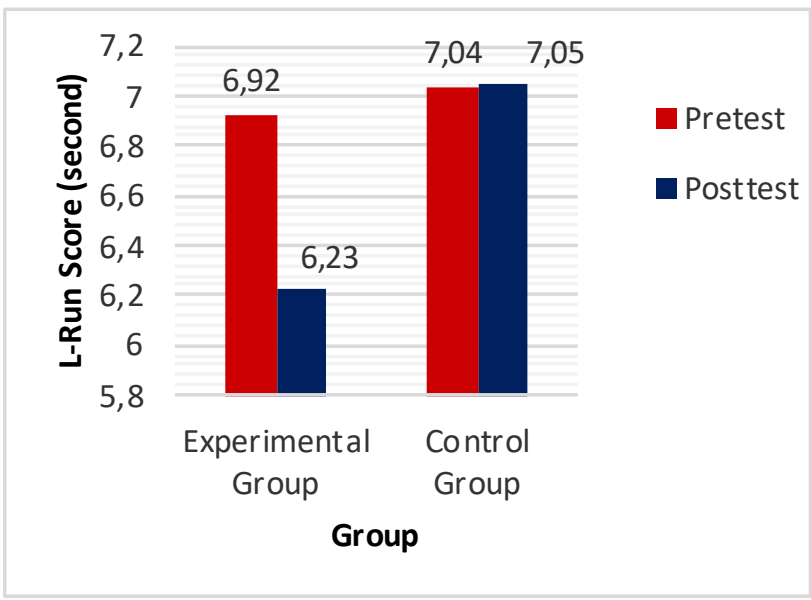

Figure 3. The Comparison Scores of Pretest and Posttest for L-Run test between and within experimental group and control group after Six Weeks of CoBAgi Training
The pretest value for L-Run test between EG and CG before six weeks of CoBAgi Training was $\mathrm{t}=0.653, \mathrm{P}=$ 4.802 indicating EG and CG were from homogenous group. After six weeks of intervention, there was significant difference between $\mathrm{EG}$ and $\mathrm{CG}$ in the posttest $\left(\mathrm{t}=-3.202, \mathrm{P}=0.003^{*}\right)$ where mean score for $\mathrm{EG}$ in the posttest was $6.23 \pm 0.69 \mathrm{sec}$. and mean score for CG was $7.05 \pm 0.74 \mathrm{sec}$. (i.e. the score difference for EG and CG was $0.82 \mathrm{sec}$.) (Table 5).

\section{Discussion}

This six weeks' study on CoBAgi training among adolescent handball players revealed the beneficial effects on coordination, dynamic balance and agility. The key findings of this study were that subjects were able to improve time taken to complete carioca and L-Run test which determine coordination and agility and also able to increase the distance reach in SEBT test which determine dynamic balance in post training testing throughout the six weeks CoBAgi Training.

There was significant improvement in time taken to complete carioca test between pre and post testing which signifies the improvement of coordination. This indicates CoBAgi training could have a beneficial effect on coordination in adolescent handball players. Coordination is a well-ordered complex motor activity of various organs and systems that necessary for high performance activity. Ricotti clarified it is relying on movement control and necessary in sports, which allow players to control their own motor actions easily and allow the learning of complex movements in a relatively rapid way [8]. Coordination improvement in adolescent is crucial and based on Yasumitsu, this will initiate rhythm skills, balance skills, transformation skills, reaction skills, consolidation skills, orientation skills and

Table 4. The Comparison of Pretest and Posttest Between and Within Control Group and Experimental Group in Carioca Score after Six Weeks with CoBAgi Training

\begin{tabular}{lllll}
\hline Group & Pre & Post & t & P \\
\hline Experimental Group & $\boldsymbol{\mu} \pm$ SD & $\boldsymbol{\mu} \pm$ SD & 2.596 & $0.015^{*}$ \\
Control Group & $10.60 \pm 2.02$ & $8.70 \pm 2.00$ & 0.389 \\
Group & & $10.11 \pm 1.33$ & 0.875 & \\
$\mathrm{t}$ & 0.046 & & & \\
$P$ & 0.964 & -2.290 & & \\
\hline
\end{tabular}

NOTE: SD - Standard Deviation; \pm , Values are presented as mean \pm standard error.

Table 5. The Comparison of Pretest and Posttest Between and Within Control Group and Experimental Group in L-Run Score after Six Weeks with CoBAgi Training

\begin{tabular}{lllll}
\hline Group & Pre & Post & t & $P$ \\
\hline Experimental Group & $\boldsymbol{\mu} \pm$ SD & $\boldsymbol{\mu} \pm$ SD & 2.680 & $0.012^{*}$ \\
Control Group & $7.04 \pm 0.74$ & $6.23 \pm 0.69$ & -0.045 & 0.964 \\
Group & & $7.05 \pm 0.74$ & & \\
t & -0.434 & -3.202 & & \\
$P$ & 0.667 & $0.003^{*}$ & & \\
\hline
\end{tabular}

NOTE: SD - Standard Deviation; \pm , Values are presented as mean \pm standard error. 
recognition skills to be translated into excellent specific skill performance [15]. Adolescent handball players would be believed to experience improvement in terms of interaction between muscles that control the movement and resulted in improved reaction skills, thus, enhanced intermuscular coordination $[11,15]$. In detail, the most contributing factors perhaps due to improvement in space-time orientation ability, kinesthetics differentiation, rhythm ability, agility and balance $[15,16]$ which being interpreted as short time taken to complete the task. It is because when player able to recognize the space within the time, prediction of action will be created cognitively and when triggered with signal, body will move rhythmically as well as balance would controlling the agility reflect [17, 18] and this how coordination take place. Considering all the facts, CoBAgi training has significantly improve coordination among adolescent handball players.

There was also significant improvement of distance reached in SEBT test between pre and post testing and this shows improvement in dynamic balance. Improvement in muscular synergies help minimize the displacement for centre of gravity in which, contribute for proper control of body balance to execute motor task [8]. This could be the reason why adolescent handball players able to maintain the stability while reaching the longer distance with only single stand. Also, the impact of improvement could reduce static postural sway and could decrease ankle or knee injuries in team handball $[11,14]$. Efficiency of this would provide positive advantage among the players. Furthermore, Integration of visual, auditory and proprioceptive (somatosensory) i.e. components of dynamic balance is capable to control body movement in space especially in sports setting [19]. Stability will reduce or even lost if stability being disturbed. Since, mechanism of dynamic balance starts when visual received the signal, it will digest the input based on stored information and interpret the demand cognitively. Initiation of movement is the result of reflection from visual input while auditory balance the body. In the meantime, proprioceptive is actually protecting the joint and muscle from excessive move which perhaps may lead to injury. According to a study, information from proprioceptors is key for conscious and unconscious control of limb movement, both for the initiation and during movements [20]. In addition, proprioceptive signals are essential for the intact neural control of movement whereas damage of proprioceptive afferents could affect the control of muscle tone, disrupts postural reflexes and severely damages spatial as well as temporal aspects of volitional movement [21]. For that, this improvement could have justified that the integration of all the components as well as performance in proprioceptive been enhanced whereas damage of it would damage the performance. Thus, apart from coordination, six weeks CoBAgi training has considerably improve dynamic balance of the adolescent players.

Apart from coordination and dynamic balance, agility also shows improvement since time taken to complete L-Run test is shorter between pre and post testing.
Favourable adaptations on endurance, muscle power, balance and strength [22] may experience by adolescent handball players. This improve their ability to be responsive such change position, direction of movement and reaction to a stimulus with speed and efficiently. Besides, move in accurate direction, and ready to change direction or stop quickly to make the play fast, smooth, efficient, and in repeatable manner [15]. Technically, this improvement was due to the re-enforcement of motor programming by neuromuscular conditioning and neural adaptation of muscle spindles, golgi-tendon organs, and joint proprioceptors [21]. These would be successful through specific learning process which imposed specificity such CoBAgi training. Responsiveness is the main part in agility and its associated with stop-and-go patterns, accelerations, decelerations, eccentric loads, changes of direction (cutting manoeuvres), and combined with demanding spatial orientation tasks [22]. Based on these facts, we may assume a successful agility requires integration of ability to react on stimulus, ability to change velocity, ability to change direction and perceptual decision making. The mechanism starts with perceptual decision making where analysis occurred cognitively, and thus it is advantage for those who has lots of information to predict on possibilities that might happen and also the corrective mechanism that could be taken. On this situation, signal already sent to the respective systems and proprioceptors activated to take charge during motor task. So that, it speeds up the information processing, thus the reflects as mentioned before could be execute quickly and safely. After all, it shows integration of neuromuscular system through neural adaptation understand such resistance as habit and thus individual able to execute motor task effectively. As suggested, intervention training with agility would enhanced neuromuscular performance as well as cognitive functioning [22].

Outcomes of present study were actually the result from combination of neuromuscular training, sport specific movement couple with perturbation which expressed as CoBAgi training. Adolescent handball players improve after six weeks of intervention. SawdonBea and Nicole stated, incorporating sport specific tasks help players/athletes to be at optimal performance [19]. To be clear, neuromuscular and sport specific training are common approach among practitioners, however, the use of perturbation is less selective and mostly implemented on stroke and post-operative knee patients. From this study, we could identify how actually perturbation would be beneficial among healthy community especially sport players. Perturbation through controlled, unpredictable, and multidirectional forces during the motor task encourage proprioceptive ability to accelerate [23]. In detailed, the challenges given on motor function e.g. quick stops, turns, changes in direction, challenges to balance and negotiating obstacles just in sudden [24]. Perhaps the best technique is without giving any visual clues. Eventually, this would alter proprioceptive (somatosensory) and make it flexible with upcoming resistance [23]. 


\section{Conclusion}

In short, the physiological adaptation was due to alteration of neural system adapted with simulated game movement i.e. sensitivity of muscle spindles increases within the muscles, so it may result in a higher readiness in responding to any destabilize forces, and this would contribute for performance and prevent excessive movement within the joint $[10,25]$. Taken together, CoBAgi training could be an alternative program to improve performance, pre-rehabilitation as well as rehabilitation for post injuries. However, the actual success factors may have limited and not be clearly defined. Thus, for further studies, the assessment should include muscle recruitment for specific components, and such effort will lead for specific program and outcome.

\section{Acknowledgements}

A special appreciation to all the participants who involve for in these study for the patience and effort to complete the tasks.

\section{Financial support}

No financial support involved.

\section{Conflict of interest}

The authors declare that no conflict of interest.

\section{References}

1. Karcher C, Buchheit M. On-court demands of elite handball, with special reference to playing positions. Sports Medicine, 2014;44(6):797-814. https://doi.org/10.1007/s40279-014-0164-z

2. Luteberget LS, Spencer M. High-intensity events in international women's team handball matches. International Journal of Sports Physiology and Performance, 20171;12(1):56-61. https://doi.org/10.1123/ijspp.2015-0641

3. Higashi RH, Santos MB, Castro GT, Ejnisman B, Sano SS, Cunha RA. Musculoskeletal injuries in young handball players: a cross-sectional study. Fisioterapia e Pesquisa, 2015;22(1):84-9.

4. Póvoas SC, Ascensão AA, Magalhães J, Seabra AF, Krustrup P, Soares JM, Rebelo AN. Analysis of fatigue development during elite male handball matches. The Journal of Strength \& Conditioning Research, 2014;28(9):2640-8. https://doi.org/10.1519/JSC.0000000000000424

5. Habelt S, Hasler CC, Steinbrück K, Majewski M. Sport injuries in adolescents. Orthop Rev (Pavia), 2011;3:18. https://doi.org/10.4081/or.2011.e18

6. Olsen OE, Myklebust G, Engebretsen L, Bahr R. Injury mechanisms for anterior cruciate ligament injuries in team handball: a systematic video analysis. The American Journal of Sports Medicine, 2004;32(4):1002-12. https://doi.org/10.1177/0363546503261724

7. Waldén M, Krosshaug T, Bjørneboe J, Andersen TE, Faul $\mathrm{O}$, Hägglund $\mathrm{M}$. Three distinct mechanisms predominate in non-contact anterior cruciate ligament injuries in male professional football players: a systematic video analysis of 39 cases. Br J Sports Med. 2015;49(22):1452-60. https://doi.org/10.1136/bjsports-2014-094573

8. Ricotti L. Static and dynamic balance in young athletes. Journal of human sport and exercise. 2011;6(4):616-28.

9. Miller MG, Herniman JJ, Ricard MD, Cheatham CC, Michael TJ. The effects of a 6-week plyometric training program on agility. Journal of Sports Science \& Medicine, 2006;5(3):459.

10.Teichmann J, Suwarganda EK, Lendewig C, Wilson BD, Yeo WK, Aziz RA, Schmidtbleicher D. Unexpected-disturbance program for rehabilitation of high-performance athletes. Journal of Sport Rehabilitation, 2016;25(2):126-32. https://doi.org/10.1123/jsr.2014-0280

11.Zech A, Hübscher M, Vogt L, Banzer W, Hänsel F, Pfeifer K. Balance training for neuromuscular control and performance enhancement: a systematic review.
Journal of Athletic Training, 2010;45(4):392-403. https://doi.org/10.4085/1062-6050-45.4.392

12.Steib S, Zahn P, zu Eulenburg C, Pfeifer K, Zech A. Time-dependent postural control adaptations following a neuromuscular warm-up in female handball players: a randomized controlled trial. BMC Sports Sci Med Rehabil, 2016;8:33. https://doi.org/10.1186/s13102-016-0058-5

13. Mansfield A, Peters AL, Liu BA, Maki BE. Effect of a perturbation-based balance training program on compensatory stepping and grasping reactions in older adults: a randomized controlled trial. Physical Therapy, 2010 Apr 1;90(4):476-91. https://doi.org/10.2522/ptj.20090070

14.Hrysomallis C. Relationship between balance ability, training and sports injury risk. Sports medicine, 2007;37(6):547-56. https://doi.org/10.2165/00007256-200737060-00007

15.Yasumitsu T, Nogawa H, Hatano Y. Effects of the Coordination Exercise Program on School Children's Agility: Short-Time Program during School Recess. ICHPER-SD Journal of Research, 2011;6(2):10-3.

16.PeerM, Salomon R, Goldberg I, BlankeO, Arzy S. Brain system for mental orientation in space, time, and person. Proceedings of the National Academy of Sciences, 2015;112(35):11072-7. https://doi.org/10.1073/pnas.1504242112

17.Magiera A, Roczniok R, Maszczyk A, Czuba M, Kantyka J, Kurek P. The structure of performance of a sport rock climber. Journal of Human Kinetics. 2013;36(1):107-17. https://doi.org/10.2478/hukin-2013-0011

18.Marczak M, Ginszt M, Gawda P, Berger M, Majcher P. Neurocognitive functioning of sport climbers. Journal of Human Kinetics, 2018;65(1):13-9. https://doi.org/10.2478/hukin-2018-0036

19.Sawdon-Bea J, Nicole S. The effects of a static and dynamic balance-training program in female volleyball players. $J$ Athl Enhanc. 2015;4: 1 . https://doi.org/10.4172/2324-9080.1000189

20.Holm I, Fosdahl MA, Friis A, Risberg MA, Myklebust $G$, Steen H. Effect of neuromuscular training on proprioception, balance, muscle strength, and lower limb function in female team handball players. Clinical Journal of Sport Medicine, 2004;14(2):88-94. https://doi.org/10.1097/00042752-200403000-00006

21.Aman JE, Elangovan N, Yeh I, Konczak J. The effectiveness of proprioceptive training for improving motor function: a systematic review. Frontiers in Human Neuroscience, 2015;28;8:1075. https://doi.org/10.3389/fnhum.2014.01075 
22.Morat M, Faude O, Hanssen H, Ludyga S, Zacher J, Eibl A, AlbrachtK, DonathL.Agility Training to Integratively Promote Neuromuscular, Cognitive, Cardiovascular and Psychosocial Function in Healthy Older Adults: A Study Protocol of a OneYear Randomized-Controlled Trial. International Journal of Environmental Research and Public Health, 2020;17(6):1853. https://doi.org/10.3390/ijerph17061853

23. Taylor JB. Lower extremity perturbation training. Strength \& Conditioning Journal. 2011;1;33(2):76-83. https://doi.org/10.1519/SSC.0b013e318211aaaa

24.Fitzgerald GK, Piva SR, Gil AB, Wisniewski SR, Oddis $\mathrm{CV}$, Irrgang JJ. Agility and perturbation training techniques in exercise therapy for reducing pain and improving function in people with knee osteoarthritis: a randomized clinical trial. Physical Therapy. 2011;91(4):452-69. https://doi.org/10.2522/ptj.20100188

25.Letafatkar A, Rajabi R, Minoonejad H, Rabiei P. Efficacy of Perturbation-Enhanced Neuromuscular Training On Hamstring and Quadriceps Onset Time, Activation and Knee Flexion During a Tuck-Jump Task. International Journal of Sports Physical Therapy, 2019;14(2): 214-227. https://doi.org/10.26603/ijspt20190214

\section{Information about the authors:}

Mohamad Hasif B. Mohamad Puzi; (Corresponding Author); Master student; https://orcid.org/0000-0002-6978-2827; acipclever@yahoo.com; Sultan Idris Education University; Malaysia.

Lee A. Choo; Associate Professor; https://orcid.org/0000-0003-4634-9083; Lee.ac@fsskj.upsi.edu.my; Sultan Idris Education University; Malaysia.

Cite this article as:

Mohamad Hasif BHP, Lee AC. The effect of six weeks CoBAgi training on coordination, dynamic balance $\&$ agility of adolescent handball players. Pedagogy of Physical Culture and Sports, 2021;25(1):31-38.

https://doi.org/10.15561/26649837.2021.0105

This is an Open Access article distributed under the terms of the Creative Commons Attribution License, which permits unrestricted use, distribution, and reproduction in any medium, provided the original work is properly cited (http://creativecommons.org/licenses/by/4.0/deed.en).

Received: 25.06 .2020

Accepted: 02.08.2020; Published: 25.02.2021 